\title{
MicroRNA in autoimmune diseases
}

Isidro Alemán-Ávila, ${ }^{1,2}$ Daniel Cadena-Sandoval, ${ }^{1}$ Mayra Jiménez Morales ${ }^{1}$ and Julián Ramírez-Bello'

${ }^{1}$ Secretaría de Salud, Hospital Juárez de México, Metabolic and Endocrine Diseases Research Unit, Ciudad de México, Mexico; ${ }^{2} I n s t i t u t o ~ P o l i t e ́ c n i c o$ Nacional, Higher School of Medicine, Program of Masters' Degree in Health Sciences

\begin{abstract}
MicroRNAs (miRNAs) are small non-coding RNAs of approximately 17-24 nucleotides in length, which complementarily and mainly bind in 3' UTR (untranslated region) regions of different messenger RNAs (mRNAs). Their general function is to negatively regulate gene expression at the posttranscriptional level, thus inhibiting translation. miRNA abnormal expression profiles have been found in different human fluids, cells and tissues affected by different autoimmune diseases, and some of them have been proposed as potential biomarkers of diagnosis, prognosis, activity, etc. in these pathologies. In addition, common variants of the human genome, called single-nucleotide polymorphisms (SNPS), located within miRNA genes, have been associated with susceptibility, severity and activity in these diseases. The purpose of this review is to describe miRNA biogenesis and function, as well as the expression profiles and SNPs in miRNA genes that are associated with different autoimmune diseases, including autoimmune thyroiditis (Hashimoto's thyroiditis and Graves' disease), systemic lupus erythematosus, rheumatoid arthritis and primary Sjögren's syndrome.
\end{abstract}

KEY WORDS: MicroRNA. Inflammation. Autoimmunity. Gene expression. Single-nucleotide polymorphisms.

\section{Introduction}

MicroRNAs (miRNAs) are small, single chain, non-coding RNAs (ncRNAs) of approximately 17 to 24 nucleotides in length, whose main function is to negatively regulate gene expression that encode proteins at the post-transcriptional level, by means of their hybridization with complementary sequences located in messenger RNA (mRNA) 3' untranslated regions (UTR), and inhibit their translation or decrease their half-life., ${ }^{1,2}$ At the cellular level, miRNAs regulate multiple normal biological processes in immune system cells, including activation/inactivation, proliferation, differentiation, apoptosis, inflammation and autoimmunity, among others. ${ }^{2-5}$ For example, miRNA-155 (miR-155) promotes inflammation by inducing T-cell activation, and miR-146a inhibits the immune response by inactivating $T$ cells; ${ }^{6}$ both effects demonstrate their participation in the regulation of the immune system, inflammation and autoimmunity, and alterations in their levels of expression contribute to the development of autoimmune diseases, such as autoimmune thyroiditis - which is divided into Hashimoto's thyroiditis and Graves' disease-, systemic lupus erythematosus (SLE), rheumatoid arthritis (RA), and primary Sjögren's syndrome (PSS). In addition, single nucleotide polymorphisms (SNPs), located in some miRNA genes, have shown an association with susceptibility for some autoimmune diseases. ${ }^{7,8}$ Owing to their different characteristics as stable molecules, wide distribution in different fluids, cells, tissues, etc. and over- or underexpression in conditions of pathology, since this expression is specific to states of disease, etc., miRNAs have been proposed as biomarkers of diagnosis, prognosis, severity and activity in various autoimmune diseases. ${ }^{9-11}$ The purpose of this review is to describe the biogenesis of miRNAs, their function, altered expression profiles and SNPs in miRNA genes
Date of reception: 20-07-2017

Date of acceptance: 18-01-2018

DOI: 10.24875/GMM.M19000233
Gac Med Mex. 2019;155:60-67

Contents available at PubMed www.gacetamedicademexico.com 


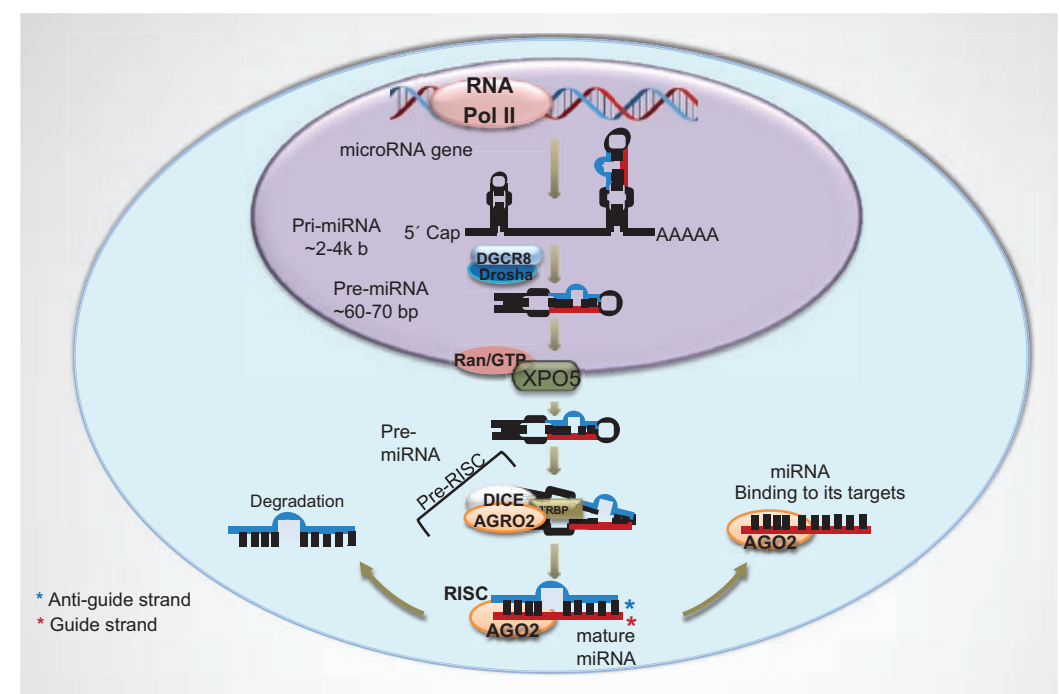

Figure 1. miRNA biogenesis. In this case, miRNAs originate in a miRNA gene with its own promoter. miRNA is mainly transcribed by the action of RNA polymerase II and general transcription factors, generating a primary miRNA (pri-miRNA) of approximately 2 to 4 kilobases. The Drosha enzyme, which has RNase III activity, generates a precursor miRNA (pre-miRNA) of approximately 70 base pairs in the cell nucleus. Subsequently, it is exported to the cytoplasm, where it is processed by the RISC complex (which contains the Dicer enzyme, also with RNase activity), generating a mature miRNA consisting of an RNA double strand; the guide strand binds to various target mRNAs, while the anti-guide strand is degraded.

associated with autoimmune thyroiditis (Graves' disease and Hashimoto's thyroiditis), SLE, RA and PSS.

\section{miRNA biogenesis}

miRNA gene expression of occurs in two forms:

- Dependent on the promoter of the gene where they reside.

- Dependent on their own promoter.

RNA polymerase II and general transcription factors are the main proteins that originate primary miRNA transcripts (pri-miRNA), which have a length of approximately 4 kilobases. ${ }^{12}$ Newly synthesized pri-miRNAs are added a Cap (7-methyl-guanosine) at their 5' end, and a polyadenine tail at their $3^{\prime}$ end. Pri-miRNAs are processed in the nucleus by the action of the "microprocessor" complex, which consists of an enzyme with RNase III activity; Drosha, and a protein with binding domains for double-stranded RNA called DGCR8 (DiGeorge syndrome chromosomal region 8, a microprocessor complex subunit). This complex is responsible for eliminating the 5' and 3 ' ends of the single stranded RNA (ss-RNA) strand from pri-miRNAs and originating precursor miRNAs (pre-miRNAs) of approximately 60 to 70 base pairs. ${ }^{12}$ Subsequently, pre-miRNAs are exported from the nucleus to the cytoplasm by the exportin 5 protein, which executes its function with energy provided by Ran-GTPase. ${ }^{13}$ In the cytoplasm, pre-miRNAs are recognized by different domains of the Dicer enzyme; the PAZ (Piwi/Argonaute/Zwile) domain has the function of recognizing and binding to double-stranded RNA (dsRNA), while the two RNase III domains cleave the pre-miRNA strand, which results in a dsRNA structure of approximately 21 base pairs. The newly generated duplex RNA interacts with a multiprotein complex known as RISC (RNA-inducing silencing complex), which is constituted of the Dicer, TRBP (transactivation-response element RNA-binding protein) and Argonaute (Ago) proteins, with the latter binding to the guide miRNA (stable) strand, while the other one is the passenger strand and is rapidly degraded (Figure 1). ${ }^{12}$

\section{miRNAs function}

In general, miRNAs bind in mRNA 3' UTR regions (although they can also bind to 5' UTRs) in two forms: by perfect complementarity and by imperfect complementarity. The proposed mechanisms for miRNA-mediated translational repression include the following:

- Translational repression. miRNAs bind to their respective mRNAs and prevent its translation. This process occurs by translation initiation inhibition, translation elongation blockage and mRNA-ribosome complex premature dissociation (Figure 2a).

- mRNA degradation. In this process, miRNAs regulate target mRNA cleavage/degradation and induce decapping and deadenylation (Figure 2b). 


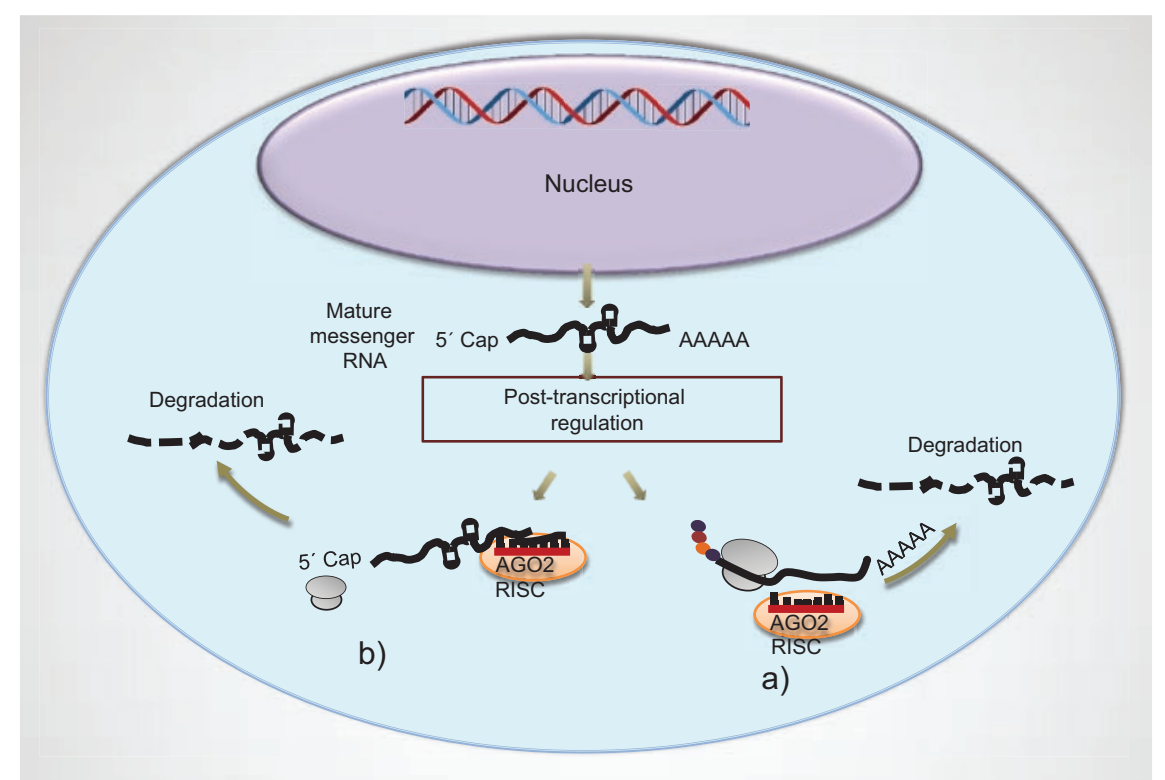

Figure 2. miRNAs function. At the posttranscriptional level, miRNAs regulate the degradation of their target mRNAs in two forms: a) at the mRNA/ ribosome level and b) by directly degrading their target $m R N A$, without going through the ribosome. The purpose in both processes is to mediate miRNAs degradation by means of their binding to mRNA 3' UTR regions, with this interaction mediating mRNA strand hydrolysis.

Finally, miRNA degradation occurs by hydrolysis, which results in an almost complete mRNA silencing. In general, miRNAs do not completely inhibit mRNAs translation, but rather significantly reduce their expression..$^{14,15}$

\section{miRNA in autoimmunity}

Various miRNAs have been associated with the onset, progression, severity, etc. of several inflammatory-autoimmune diseases because they regulate key points of the innate-adaptive immune response. For example, miR-146a negatively regulates the synthesis of various pro-inflammatory cytokines and the proliferation of immune system cells. ${ }^{16}$ Furthermore, in murine models, its deletion is associated with chronic deregulation of the nuclear factor-kappa B (NF-kB) pathway. Different studies have shown that this miRNA targets the mRNA 3' UTRs: IRAK-1 (interleukin 1 receptor associated kinase 1), STAT1 (signal transducer and activator of transcription 1) and TRAF6 (TNF receptor-associated factor 6), among others (cell signaling proteins involved in inflammation/ autoimmunity) (Figure 3).

Thus miR-146a underexpression does not effectively downregulate IRAK1, STAT1, TRAF1 expression; consequently, there is an increase in tumor necrosis factor alpha (TNF- $\alpha$ ) and IL-1 $\beta$ cytokines (Figure 3), among others. ${ }^{10,17}$ Knock-out of this gene entails the loss of negative regulation of type $\mathrm{I}$ interferon synthesis negative regulation in plasmacytoid dendritic cells and predisposes to autoimmune phenotypes. ${ }^{18,19}$

In T cells, the deletion of Dicer (an enzyme that generates miRNA mature forms) at an early differentiation stage generates a reduction in the number of $T$ cells in peripheral lymphoid organs and the thymus. ${ }^{10,20}$ At $B$-cell early stages, the same deletion resulted in a blockage of the pro- to pre-B cell transition. ${ }^{10}$ In addition, Dicer knock-out in murine models generates an increase in autoantibody titers and immune complexes deposits in specific sections of the kidney. ${ }^{10}$ Other studies indicate that deletion or overexpression of certain miRNAs result in impaired B cell tolerance and accelerate the development of autoimmune diseases. ${ }^{19-21}$ These data indicate that miRNAs play a critical role in B-T lymphocytes selection and in the maintenance of immune tolerance to self-antigens. ${ }^{20}$

On the other hand, different genetic studies have shown that SNPs located in some miRNA genes contributes with susceptibility to develop various autoimmune diseases. ${ }^{22,23}$

\section{miRNA in autoimmune thyroiditis}

Autoimmune thyroiditis is characterized for being an organ-specific autoimmune thyroid disease and is divided into Graves' disease and Hashimoto's thyroiditis. ${ }^{24}$ Different studies have identified miRNA altered expression profiles in patients with autoimmune thyroiditis. 


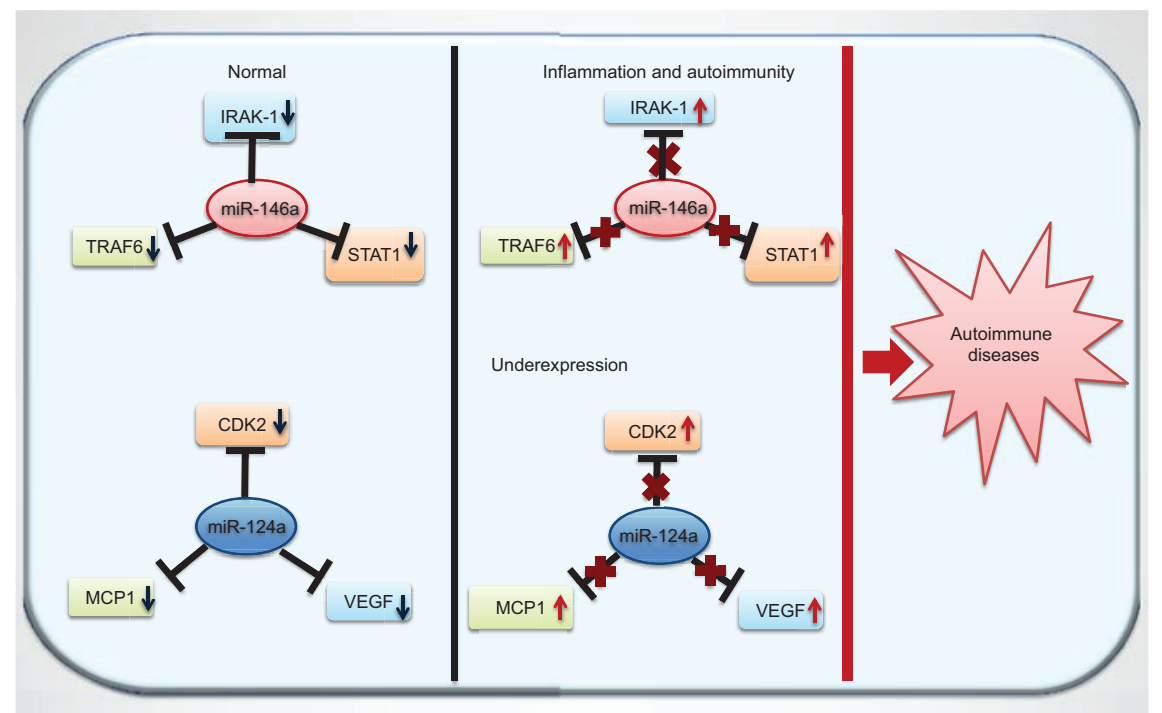

Figure 3. Biological effect of miR-146a and miR-24-3p underexpression in autoimmune diseases. Underexpression of both miRNAs (IRF5, IRAK1, TRAF6, STAT1, CDK2, MCP1, VEGF, etc. inhibitors) entails a higher amount of IRF5, IRAK1, TRAF6, STAT1, MCP1, VEGF, etc. mRNA/ proteins, which are proteins involved in inflammation and autoimmunity.

Graves' disease is caused by an excessive production of autoantibodies directed against various thyroid antigens; one of them, which also represents the main autoantigen, is the thyroid-stimulating hormone receptor. Autoantibodies against this receptor stimulate and cause this gland to produce a larger amount of thyroid hormones such as triiodothyronine (T3) and thyroxine (T4), which results in autoimmune hyperthyroidism. ${ }^{25}$

Recent studies have identified elevated levels of the following miRNAs in serum from patients with Graves' disease: miR-16, miR-22, miR-375 and miR-451, among others $^{26}$ (Table 1). Under normal conditions, miR-16 negatively regulates IKK $\alpha$ (kappa-B kinase subunit alpha) kinase expression; therefore, its underexpression during macrophage differentiation is correlated with an IKK $\alpha$ increase, which phosphorylates NF-kB and activates inflammation-related pathways. ${ }^{27}$ Thus far, it is not understood why this miRNA is overexpressed in serum of patients with Graves' disease,;6 further studies are necessary to understand this finding.

On the other hand, miR-154, miR-376b and miR-431 have been found to be underexpressed in peripheral blood mononuclear cells (PBMC) of patients with newly diagnosed Graves' disease (Table 1), whereas in patients with Graves' disease in remission they are expressed again. Therefore, underexpression of these miRNAs in PBMC might induce an inflammatory response in this pathology. ${ }^{28}$ On the other hand, patients with Graves' disease who have been treated for years and continue to be positive for autoantibodies have shown serum miR-23b-5p and miR-92a-39 lower levels of expression in comparison with patients, while miRNA let-7g-3p and miR-339-5p showed overexpression (Table 1). For this reason, these ncRNAs have been proposed as biomarkers to monitor or predict Graves' disease activity. ${ }^{29}$

Some SNPs located in miRNA genes have been associated with this disease. One of them, the miR$499 \mathrm{rs} 3746444 \mathrm{~A} / \mathrm{G}$ polymorphism, located at the pre-miRNA level, affects not only its processing but also its expression and binding to its target mRNAs. ${ }^{30}$ Recently, the miR-499 rs3746444 A/G genotype was found to be associated with susceptibility to Graves' disease (OR $=1.32, p=0.013$ ) (Table 2). One of miR499 targets is the IL-17 receptor (IL-17RB). Reduction in the expression of this miRNA leads to an IL-17RB and IL-17 increase, with the latter playing a determining role in the pathophysiology of various autoimmune diseases. ${ }^{31}$ On the other hand, Inoue et al. showed that the miR-125a rs12976445C/T polymorphism confers susceptibility to Graves' disease. ${ }^{32}$

On the other hand, Hashimoto's thyroiditis, also known as autoimmune hypothyroidism, is caused by thyroid gland invasion by lymphoid cells, which cause inflammation, gradual atrophy of the thyroid tissue and interfere with its ability to produce thyroid hormones, which results in hypothyroidism. ${ }^{33}$

Regarding the role of miRNAs in Hashimoto's thyroiditis, one study identified miR-22, miR-375 and miR-451 overexpressed serum levels (Table 1). ${ }^{26} \mathrm{Un}$ der normal conditions, miR-22 inhibits estrogen receptor alpha $(E R \alpha)$ estrogen-mediated signaling and 
Table 1. Altered miRNA expression profiles in patients with autoimmune diseases

\begin{tabular}{|c|c|c|c|c|}
\hline \multirow[t]{2}{*}{ Disease } & \multirow[t]{2}{*}{ Sample } & \multicolumn{2}{|r|}{ Expression } & \multirow[t]{2}{*}{ References } \\
\hline & & Underexpressed & Overexpressed & \\
\hline \multirow[t]{2}{*}{ GD } & Serum & & $\begin{array}{l}\text { miR-16, miR-22, } \\
\text { miR-375, miR-451 }\end{array}$ & 27 \\
\hline & PBMC & miR-154, miR-376b, miR-431 & & 28 \\
\hline \multirow[t]{2}{*}{ HT } & Serum & & $\begin{array}{l}\text { miR-22, miR-375, miR-451 } \\
\text { miR-let-7e }\end{array}$ & 26 \\
\hline & PBMC & miR-125a-3p & & 34,35 \\
\hline \multirow[t]{2}{*}{ SLE } & PBMC & $\begin{array}{l}\text { miR-196a, miR-17-5p, } \\
\text { miR-127-3p, miR-410 }\end{array}$ & $\begin{array}{l}\text { miR-21, miR-61, miR-78, miR-126, miR-142-3p, } \\
\text { miR-148a }\end{array}$ & 40,41 \\
\hline & Serum/plasma & $\begin{array}{l}\text { miR-125a, miR-130b-3p, } \\
\text { miR-17-5p, miR-112, miR-196a, } \\
\text { miR-155 }\end{array}$ & miR-223, miR-21, miR-126, miR-223 & $36-41$ \\
\hline \multirow[t]{3}{*}{ RA } & Synovial fibroblasts & $\begin{array}{l}\text { miR-124a, miR-34a } \\
\text { miR-21 }\end{array}$ & $\begin{array}{l}\text { miR-221, miR-146a, } \\
\text { miR-146b }\end{array}$ & 46,48 \\
\hline & PBMC & $\begin{array}{l}\text { miR-16, miR-125a-3p, miR-126-3p, } \\
\text { miR-132, }\end{array}$ & miR-16, miR-21, miR-24, miR-26a, miR-125a-5p & 46 \\
\hline & Plasma/serum & miR-146a & & 46 \\
\hline \multirow[t]{2}{*}{ PSS } & $\begin{array}{l}\text { PBMC } \\
\text { Salivary glands }\end{array}$ & & miR-146a, miR-155 & $51-52$ \\
\hline & $\begin{array}{l}\text { Salivary gland } \\
\text { tissue }\end{array}$ & & miR-16, miR-181a & 53 \\
\hline
\end{tabular}

GD = Graves' disease, $\mathrm{HT}=$ Hashimoto's thyroiditis, $\mathrm{SLE}=$ systemic lupus erythematosus, RA = rheumatoid arthritis, $\mathrm{PSS}=$ primary Sjögren's syndrome, $\mathrm{PBMC}=$ peripheral blood mononuclear cells.

expression. Overexpression of this miRNA results in the repression of estrogen-ER $\alpha$ signaling pathway, which is required for $T$ cells differentiation; therefore, its overexpression affects this process. ${ }^{27}$ Another miRNA that has shown overexpression in PBMC of patients with Hashimoto's thyroiditis is miR-let-7e, whereas miR-125a-3p has been found to be underexpressed $^{34,35}$ (Table 1).

In relation to the association between miRNA genetic variants and Hashimoto's thyroiditis, few studies have been conducted: two showed that the $\mathrm{rs} 12976445 \mathrm{C} / \mathrm{T}$ SNP located in the primiR-125a sequence confers protection for this pathology $(\mathrm{OR}=0.53, \mathrm{p}=0.027)$ (Table 2). ${ }^{30,32}$

\section{miRNA in SLE}

SLE represents the prototype of autoimmune diseases. It is characterized for affecting any organ, tissue or system due to the attack of different autoantibodies directed to nuclear or cytoplasmic self-antigens, such as DNA, lipids, ribonucleoproteins, etc. A key cytokine in SLE is type I interferon, which is
Table 2. Studies of SNPs in miRNA genes and susceptibility to autoimmune diseases

\begin{tabular}{lccccc}
\hline Disease & miRNA & SNP & OR & p & Reference \\
\hline GD & miR-499 & rs3746444A/G & 1.32 & 0.013 & 31 \\
HT & miR-125a & $r s 12976445 \mathrm{C} / \mathrm{T}$ & 0.53 & 0.027 & 32 \\
SLE & miR-146a & $\mathrm{rs} 57095329 \mathrm{~A} / \mathrm{G}$ & 1.29 & 0.0001 & 41 \\
& & $\mathrm{rs} 24311697 \mathrm{C} / \mathrm{T}$ & 1.49 & 0.001 & 41 \\
$\mathrm{RA}$ & $\mathrm{miR}-499$ & $\mathrm{rs} 3746444 \mathrm{~A} / \mathrm{G}$ & 3.88 & 0.002 & 49 \\
\hline
\end{tabular}

$\mathrm{SNP}=$ single nucleotide polymorphisms, $\mathrm{OR}=$ odds ratio, $\mathrm{GD}=$ Graves' disease,

$H T=$ Hashimoto's thyroiditis, SLE = systemic lupus erythematosus, RA = rheumatoid arthritis

involved with a broad range of clinical traits and activity thereof. ${ }^{36}$

The most commonly referred miRNAs in PBMC, serum, plasma or $\mathrm{T}$ cells and that are overexpressed in SLE are miR-21, miR-61, miR-78, miR-126, miR142-3p, miR-148a, miR-189, miR-410, miR-423-5p (Table 1), whereas miR-146a, miR-125a, miR-155, miR-130b-3p, miR-17-5p, miR-196a, miR-184, etcetera, have been identified to be underexpressed ${ }^{10,37-40}$ (Table 1). Under normal conditions, miR-126 binds to the DNA methyltransferase 1 enzyme mRNA, and its overexpression leads to a decrease of this enzyme, 


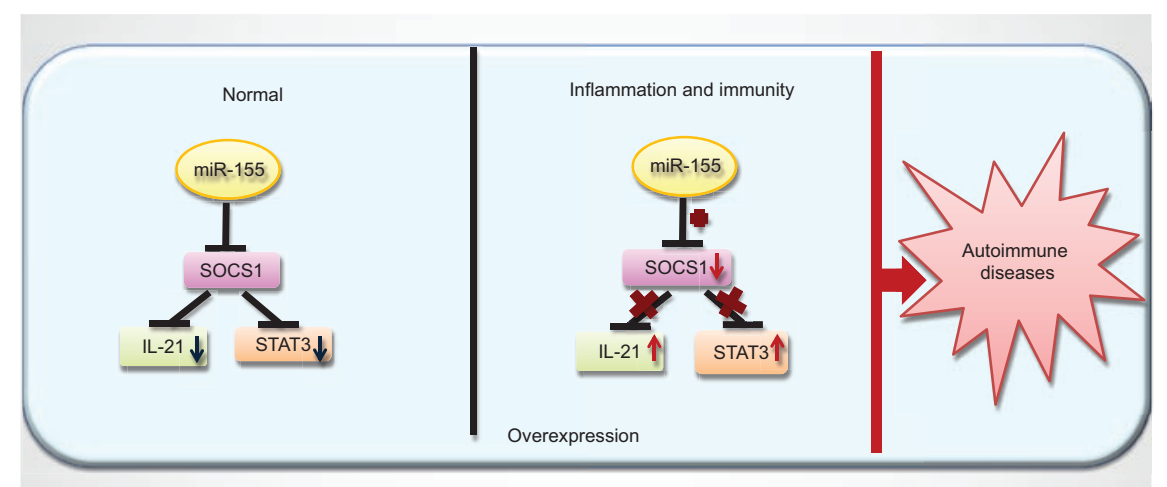

Figure 4. miR-155 overexpression biological effect in autoimmune diseases. miR-155 overexpression leads to a lower amount of its target mRNA: SOCS1. This way, this event leads to SOCS1 lower expression and IL-21 and STAT3 higher expression.

which causes hypomethylation in binding regions for this protein and a higher expression of CD11a and CD70 mRNA/proteins, which are important in the pathogenesis of SLE. ${ }^{40}$ On the other hand, under normal conditions, miR-155 inhibits suppressor of cytokine signaling 1 (SOCS1) expression, which regulates STAT3 phosphorylation. A recent study in PBMC of patients with SLE identified underexpression of this miRNA, which generated an increase in SOCS1 expression; in the same study, miR-155 was overexpressed, which generated a decrease in SOCS1 and an increase in STAT3 phosphorylation, as well as in IL-21 function (Figure 4). In the context of autoimmunity, this cytokine acts as a guardian gene for the proliferation and differentiation of $B$ cells towards autoantibody-generating plasma cells. This way, larger amounts of IL-21 (together with other cytokines) induce antigen-independent B-cell proliferation and increase BCR-mediated signaling. ${ }^{41}$

Two studies reported SNPs in miR-146a that are associated with SLE. The rs57095329A/G SNP, located in the miR-146a promoter, showed association with susceptibility to SLE in Asians (OR $=1.29, p=0.0001$ ) (Table 2). ${ }^{40}$ In addition, the rs24311697C/T SNP, located between the gene that encodes for the pituitary tumor transforming protein 1 and miR-146a, also showed an association with susceptibility to SLE (T allele, $\mathrm{OR}=1.23, \mathrm{p}=0.001$; $\mathrm{TT}$ genotype, $\mathrm{OR}=1.49$, $\mathrm{p}=0.001$ ) (Table 2). ${ }^{41}$ On the other hand, other studies indicate that both the rs2910164G/C SNP located in pre-miR-146a and miR-499 rs3746444A/G do not confer susceptibility to SLE. ${ }^{42,43}$

\section{miRNA in RA}

RA is a systemic, inflammatory, chronic autoimmune disease characterized by inflammation of the synovial membranes with joint shifting and destruction, which causes deformity, disability of the affected individual and can be accompanied by different extra-articular manifestations. ${ }^{23}$

Several studies have shown miRNA altered expression patterns in PBMC, T lymphocytes, synovial fibroblasts, osteoclasts, synovial fluid, etc., of patients with RA. ${ }^{44,45} \mathrm{miR}-146 \mathrm{a} / \mathrm{b}$ have been found to be overexpressed in different cell types, such as PBMC, synovial fibroblasts, synovial tissue and fluid, etc. ${ }^{45}$ These data should be taken with caution since, in SLE and in murine models, miR-146a deletion or underexpression entail the generation of spontaneous autoimmunity or contribute to the development of SLE, respectively. ${ }^{10,19}$ It is difficult to determine a similar role in different fluids and cells of patients with RA, given that this miRNA has been found to be overexpressed and not underexpressed. Further studies are needed to determine their role in AR. Other miRNAs that are overexpressed in plasma, synovial fluid/tissue, PBMC or synovial fibroblasts of patients with RA are miR-16, miR-21, miR-24, miR-26a, miR-125b, miR-126-3p, miR-155, miR-221, miR-222 and miR-451 (Table 1); miR-124a, miR-125a-3p, miR-126-3p, miR-132 and miR-155 have been found to be underexpressed ${ }^{44,45}$ (Table 1). miR-124a regulates the expression of cyclin-dependent kinase-2 (CDK2), monocyte chemoattractant protein 1 (MCP1) and vascular endothelial growth factor (VEGF). Thus, according to its targets, miR-124a regulates proliferation, leukocyte chemoattraction and angiogenesis, respectively. Therefore, its decrease leads to CDK-2, MCP-1 and VEGF higher expression ${ }^{46}$. In turn, overexpressed miR-221 in synovial fibroblasts increases the synthesis of pro-inflammatory cytokines, promotes migration and resistance to apoptosis. ${ }^{47}$ 
As regards genetic susceptibility in RA, an association with the miR-499 rs3746444A/G SNP has been reported: AA vs. $G G$ genotype, $O R=3.88, p=0.002$, but not of the miR-146a rs2910164G/C SNP, in Iranians $^{48}$ (Table 2). However, another study showed that the miR-499 rs3746444A/G polymorphism is associated with protection for RA (AA vs. $A G, O R=0.3, p$ $=0.005$ ) (Table 2), disease activity and toxicity for methotrexate in an Egyptian population. The miR196a2 rs11614913 SNP showed no association with RA in the same population. ${ }^{49}$

\section{miRNA in PSS}

Primary Sjögren's syndrome (PSS) is a chronic autoimmune disease characterized by inflammation, lymphocytic infiltration of exocrine glands, and especially by involvement of the buccal (xerostomia) and ocular glands (xerophthalmia), which causes dryness. PSS can also affect organs and systems such as the kidneys, the liver, as well as the nervous and circulatory systems. ${ }^{50}$

Altered miRNA gene expression profiles have been identified in PSS. Two studies have identified miR146a overexpression in PBCM. ${ }^{51,52}$ On the other hand, Gourzi et al. suggested that miRNAs 16, 181a, 200b, $200 b^{*}, 23$ and $483-5 p$ can predict a poor prognosis in this disease (Table 1). ${ }^{53}$ miR-16 and miR-181a high expression has been associated with a decrease in minor salivary glands function. ${ }^{53} \mathrm{~A}$ recent study showed 25 different miRNAs that were overexpressed and one under-expressed in patients with PSS in comparison with controls; those that were overexpressed include let-7e-5p, miR-16-1-3p, miR-20b-5p, miR-21, miR-146a, miR-424-3p, miR-190a, miR-21-3p, among others, while miR-150-5p was found to be underexpressed..$^{54}$ Regarding genetic susceptibility in PSS, thus far there are no reported studies of polymorphisms in miRNA genes.

\section{Conclusions}

miRNAs potentially represent new therapeutic targets of interest in various medical areas, where different autoimmune diseases are included. miRNAs importantly contribute in the regulation of all normal and pathological processes involved in the immune system and include cytokine activation/inactivation, proliferation, differentiation, synthesis, etc. Altered expression profiles of various miRNAs have been identified in Graves' disease, Hashimoto's thyroiditis, RA, SLE and PSS, among others. Several of them have shown under- or overexpression, which has been associated with the development, poor prognosis, severity, etc. of these conditions. In addition, located in miRNA genes have shown an association with susceptibility to develop different autoimmune diseases. However, few studies have been carried out in various populations. Therefore, it is necessary to evaluate these variants in miRNA genes in different autoimmune diseases and populations. Despite all data generated in these pathologies, we must take the results with caution, given that several studies show controversial data. It is important to continue studying the role of these miRNAs and in more detail in these pathologies in order to determine their roles as possible therapeutic targets or prognostic biomarkers.

\section{Acknowledgements}

The authors are grateful for the financing granted by CONACyT (FOSISS, project 233107). Isidro Alemán-Ávila thanks CONACyT for grant 651960, support number 453284, granted during the completion of his master's degree.

\section{References}

1. Mendell JT. MicroRNAs: critical regulators of development, cellular physiology and malignancy. Cell Cycle. 2005;4:1179-1184.

2. Ambros V. The functions of animal microRNAs. Nature. 2004;431: 350-355.

3. Bernecker C, Lenz L, Ostapczuk MS, Schinner S, Willenberg H, Ehlers M, et al. MicroRNAs miR-146a1, miR-155 2, and miR-200a1 are regulated in autoimmune thyroid diseases. Thyroid. 2012;22:1294-1295.

4. Baltimore D, Boldin MP, O'Connell RM, Rao DS, Taganov KD. MicroRNAs: new regulators of immune cell development and function. Nat Immunol. 2008;9:839-845.

5. O'Connell RM, Rao DS, Chaudhuri AA, Baltimore D. Physiological and pathological roles for microRNAs in the immune system. Nat Rev Immunol. 2010;10:111-122.

6. Li K, Du Y, Jiang BL, He JF. Increased microRNA-155 and decreased microRNA-146a may promote ocular inflammation and proliferation in Graves' ophthalmopathy. Med Sci Monit. 2014;20:639-643.

7. Liu X, Han Z, Yang C. Associations of microRNA single nucleotide polymorphisms and disease risk and pathophysiology. Clin Genet. 2016;92:235-242.

8. Chatzikyriakidou A, Voulgari PV, Georgiou I, Drosos AA. miRNA and related polymorphisms in rheumatoid arthritis susceptibility. Autoimmun Rev. 2012;11:636-641.

9. Alevizos I, Illei GG. MicroRNAs as biomarkers in rheumatic diseases. Nat Rev Rheumatol. 2010;6:391-398.

10. Chen JQ, Papp G, Szodoray P, Zeher M. The role of microRNAs in the pathogenesis of autoimmune diseases. Autoimmun Rev. 2016;15: 1171-1180.

11. Marques-Rocha JL, Samblas M, Milagro Fl, Bressan J, Martínez JA, Marti A. Noncoding RNAs, cytokines, and inflammation-related diseases. FASEB J. 2015;29:3595-3611.

12. UI Hussain M. Micro-RNAs (miRNA): genomic organisation, biogenesis and mode of action. Cell Tissue Res. 2012;349:405-413.

13. Bohnsack MT, Czaplinski K, Gorlich D. Exportin 5 is a RanGTP-dependent dsRNA-binding protein that mediates nuclear export of pre-miRNA. RNA. 2004;10:185-191.

14. Cannell IG, Kong YW, Bushell M. How do microRNAs regulate gene expression? Biochem Soc Trans. 2008;36:1224-1231. 
15. Mohr AM, Mott JL. Overview of microRNA biology. Semin Liver Dis. 2015;35:3-11.

16. Lee HM, Kim TS, Jo EK. MiR-146 and miR-125 in the regulation of innate immunity and inflammation. BMB Rep. 2016;49:311-318.

17. Tang $Y$, Luo $X$, Cui $H, N i X$, Yuan M, Guo $Y$, et al. MicroRNA-146a contributes to abnormal activation of the type I interferon pathway in human lupus by targeting the key signaling proteins. Arthritis Rheum. 2009;60:1065-1075.

18. Boldin MP, Taganov KD, Rao DS, Yang L, Zhao JL, Kalwani M, et al. miR-146a is a significant brake on autoimmunity, myeloproliferation, and cancer in mice. J Exp Med. 2011;208:1189-1201.

19. Garo LP, Murugaiyan G. Contribution of microRNAs to autoimmune diseases. Cell Mol Life Sci. 2016;73:2041-2051.

20. Cobb BS, Nesterova TB, Thompson E, Hertweck A, O'Connor E, Godwin J, et al. T cell lineage choice and differentiation in the absence of RNase III enzyme Dicer. J Exp Med. 2005;201:1367-1373.

21. González-Martín A, Adams BD, Lai M, Shepherd J, Salvador-Bernaldez M, Salvador JM, et al. The microRNA miR-148a functions as a critical regulator of B cell tolerance and autoimmunity. Nat Immunol. 2016;17: 433-440.

22. Ramírez-Bello J, Jiménez-Morales S. Implicaciones funcionales de los polimorfismos (SNP) en genes codificantes de proteínas y no codificantes en enfermedades multifactoriales. Gac Med Mex. 2017;153:238-250.

23. Rodríguez-Elías AK, Maldonado-Murillo K, López-Mendoza LF Ramírez-Bello J. Genetics and genomics in rheumatoid arthritis (RA): an update. Gac Med Mex. 2016;152:218-227.

24. Brown RS. Autoimmune thyroid disease: unlocking a complex puzzle. Curr Opin Pediatr. 2009;21:523-528

25. Burch HB, Cooper DS. Management of Graves disease: a review. JAMA 2015;314:2544-2554

26. Yamada $\mathrm{H}$, Itoh $\mathrm{M}$, Hiratsuka I, Hashimoto S. Circulating microRNAs in autoimmune thyroid diseases. Clin Endocrinol (Oxf). 2014;81:276-281.

27. Li T, Morgan MJ, Choksi S, Zhang Y, Kim YS, Liu ZG. MicroRNAs modulate the noncanonical transcription factor NF-kappaB pathway by regulating expression of the kinase IKKalpha during macrophage differentiation. Nat Immunol. 2010;11:799-805.

28. Liu R, Ma X, Xu L, Wang D, Jiang X, Zhu W, et al. Differential microRNA expression in peripheral blood mononuclear cells from Graves' disease patients. J Clin Endocrinol Metab. 2012;97:E968-E972.

29. Hiratsuka I, Yamada H, Munetsuna E, Hashimoto S, Itoh M. Circulating microRNAs in Graves' disease in relation to clinical activity. Thyroid. 2016;26:1431-1440.

30. Cai T, Li J, An X, Yan N, Li D, Jiang Y, et al. Polymorphisms in MIR499a and MIR125a gene are associated with autoimmune thyroid diseases. Mol Cell Endocrinol. 2017;440:106-115

31. Singh RP, Hasan S, Sharma S, Nagla S, Yamaguchi DT, Wong DT, et al. Th17 cells in inflammation and autoimmunity. Autoimmun Rev. 2014;13:1174-1181.

32. Inoue $\mathrm{Y}$, Watanabe M, Inoue N, Kagawa T, Shibutani S, Otsu H, et al Associations of single nucleotide polymorphisms in precursor-microRNA (miR)-125a and the expression of mature miR-125a with the development and prognosis of autoimmune thyroid diseases. Clin Exp Immunol. 2014:178:229-235.

33. Pyzik A, Grywalska E, Matyjaszek-Matuszek B, Roliński J. Immune disorders in Hashimoto's thyroiditis: what do we know so far? J Immunol Res. 2015;2015:979167.

34. Kagawa T, Watanabe M, Inoue N, Otsu H, Saeki M, Katsumata $Y$, et al. Increases of microRNA let-7e in peripheral blood mononuclear cells in Hashimoto's disease. Endocr J. 2016;63:375-380.

35. Peng H, Liu Y, Tian J, Ma J, Tang X, Yang J, et al. Decreased expression of microRNA-125a-3p upregulates interleukin-23 receptor in patients with Hashimoto's thyroiditis. Immunol Res. 2015;62:129-136.

36. Zan H, Tat C, Casali P. MicroRNA in lupus. Autoimmunity. 2014;47: 272-285.
37. Yan S, Yim LY, Lu L, Lau CS, Chan VS. MicroRNA regulation in systemic lupus erythematosus pathogenesis. Immune Netw. 2014;14: 138-148.

38. Pan W, Zhu S, Yuan M, Cui H, Wang L, Luo X, et al. MicroRNA-21 and microRNA-148a contribute to DNA hypomethylation in lupus CD4+ T cell by directly and indirectly targeting DNA methyltransferase 1 . J Immunol. 2010;184:6773-6781.

39. Tang ZM, Fang M, Wang JP, Cai PC, Wang P, Hu LH. Clinical relevance of plasma miR-21 in new-onset systemic lupus erythematosus patients. $\mathrm{J}$ Clin Lab Anal. 2014;28:446-451.

40. Husakova M. MicroRNAs in the key events of systemic lupus erythematosus pathogenesis. Biomed Pap Med Fac Univ Palacky Olomouc Czech Repub. 2016;160:327-342.

41. Rasmussen TK, Andersen T, Bak RO, Yiu G, Sørensen CM, Stengaard-Pedersen $\mathrm{K}$, et al. Overexpression of microRNA-155 increases IL-21 mediated STAT3 signaling and IL-21 production in systemic lupus erythematosus. Arthritis Res Ther. 2015;17:154.

42. Lee YH, Bae SC. The miR-146a polymorphism and susceptibility to systemic lupus erythematosus and rheumatoid arthritis: a meta-analysis. Z Rheumatol. 2015;74:153-156.

43. Fu L, Jin L, Yan L, Shi J, Wang H, Zhou B, et al. Comprehensive review of genetic association studies and meta-analysis on miRNA polymorphisms and rheumatoid arthritis and systemic lupus erythematosus susceptibility. Hum Immunol. 2016;77:1-6.

44. Murata K, Furu M, Yoshitomi H, Ishikawa M, Shibuya $H$, Hashimoto M, et al. Comprehensive microRNA analysis identifies miR-24 and miR$125 a-5 p$ as plasma biomarkers for rheumatoid arthritis. PLoS One. 2013;8:e69118.

45. Churov AV, Oleinik EK, Knip M. MicroRNAs in rheumatoid arthritis: altered expression and diagnostic potential. Autoimmun Rev. 2015;14:1029-1037.

46. Nakamachi $Y$, Kawano S, Takenokuchi M, Nishimura K, Sakai Y, Chin T, et al. MicroRNA-124a is a key regulator of proliferation and monocyte chemoattractant protein 1 secretion in fibroblast-like synoviocytes from patients with rheumatoid arthritis. Arthritis Rheum. 2009;60: 1294-1304.

47. Yang S, Yang Y. Downregulation of microRNA-221 decreases migration and invasion in fibroblast-like synoviocytes in rheumatoid arthritis. Mol Med Rep. 2015;12:2395-2401.

48. Hashemi M, Eskandari-Nasab E, Zakeri Z, Atabaki M, Bahari G, Jahantigh M, et al. Association of pre-miRNA-146a rs2910164 and pre-miR-499 rs3746444 polymorphisms and susceptibility to rheumatoid arthritis. Mol Med Rep. 2013;7:287-291.

49. Toraih EA, Ismail NM, Toraih AA, Hussein MH, Fawzy MS. Precursor miR499a variant but not miR-196a2 is associated with rheumatoid arthritis susceptibility in an Egyptian population. Mol Diagn Ther. 2016;20: 279-295.

50. Alevizos I, Illei GG. MicroRNAs in Sjögren's syndrome as a prototypic autoimmune disease. Autoimmun Rev. 2010;9:618-621.

51. Zilahi E, Tarr T, Papp G, Griger Z, Sipka S, Zeher M. Increased microRNA-146a/b, TRAF6 gene and decreased IRAK1 gene expressions in the peripheral mononuclear cells of patients with Sjögren's syndrome. Immunol Lett. 2012;141:165-168.

52. Pauley KM, Stewart CM, Gauna AE, Dupre LC, Kuklani R, Chan AL et al. Altered miR-146a expression in Sjögren's syndrome and its functional role in innate immunity. Eur J Immunol. 2011;41:2029-2039.

53. Gourzi VC, Kapsogeorgou EK, Kyriakidis NC, Tzioufas AG. Study of microRNAs (miRNA) that are predicted to target the autoantigens Ro/ SSA and La/SSB in primary Sjögren's Syndrome. Clin Exp Immunol. 2015;182:14-22.

54. Chen JQ, Papp G, Póliska S, Azabó K, Tarr T, Bálint BL, et al. MicroRNA expression profiles identify disease-specific alterations in systemic lupus erythematosus and primary Sjögren】s syndrome. PLoS One. 2017;12: e0174585. 